\title{
Near-Infrared Fluorescence Imaging
}

National Cancer Institute

\section{Source}

National Cancer Institute. Near-Infrared Fluorescence Imaging. NCI Thesaurus. Code C126402.

Fluorescence imaging using dyes that fluoresce in the near-infrared range. NIR optical imaging permits relatively deep photon penetration into tissue, minimal tissue autofluorescence, less scatter, and high optical contrast. 